\title{
Pulmonary sarcoidosis mimicking multiple pulmonary metastases
}

\author{
M. Majori1, M. Anghinolfi1, A. Scarascia2, L. Gnetti3, A. Pavarani4, A. Casalini1
}

An asymptomatic 67-year-old man was referred to our attention for a second opinion regarding the aetiology of multiple pulmonary nodules in May 2009.

The patient had a history of surgery for colorectal cancer 30 years ago. Multiple pulmonary nodules had first been noted in August 2008 during cancer long-term follow-up (fig. 1a).

Imaging characteristics of the nodules resembled those of pulmonary metastases, and the patient underwent a F-18 fluorodeoxyglucose (FDG) positron emission tomography (PET) to characterise the nodules and to detect possible lesions of a primary malignancy. PET excluded FDG uptake in the pulmonary nodules; however, there was a hypermetabolic lesion suspicious of a primary malignancy seen in the thyroid. Fine-needle aspiration biopsy was indicative of a thyroid cancer, although the findings were not definitive with a small sample size. A biopsy from pulmonary nodules was not performed. The patient was diagnosed with thyroid cancer with lung metastases and underwent total thyroidectomy. However, the diagnosis was changed to thyroid adenoma after pathological examination.

After this unsuccessful work-up, the patient was referred to our hospital for further evaluation of multiple pulmonary nodules. The patient's conditions were good. His physical examination and laboratory findings that included determination of levels of tumour markers were unremarkable. Results of a pulmonary function test and arterial blood gas in room air were normal. A thin-section CT scan did not show any nodules changes (fig. $1 \mathrm{~b}$ ).

The initial presumptive diagnosis of multiple pulmonary metastases was revised after meticulous medical history revealed antiviral therapy for chronic hepatitis $\mathrm{C}$ virus (HCV) infection from February 2008 to May 2008.

Bronchoscopy with transbronchial lung biopsy (TBLB) was performed. Histopathologic examination showed non-necrotising sarcoidal granulomas with many multinucleated giant cells (fig. 2). Histochemical stains and microbiologic cultures for fungi and mycobacteria were negative.

The patient was diagnosed with interferoninduced sarcoidosis, remains asymptomatic, and his disease had neither improved nor progressed. As he had no past medical history of sarcoidosis or radiological signs of sarcoidosis before IFN therapy, we evaluated the case as the novo.

The first recognised case of IFN-associated sarcoidosis was reported in 1987 in a women treated with IFN- $\alpha$ for renal cell carcinoma [1]. Since then, numerous cases have been published, suggesting a relationship between sarcoidosis and interferon treatment in patients with a variety of disease including chronic hepatitis C. Moreover, HCV infection, by itself, has been related to the development of sarcoidosis, in the absence of IFN therapy [2, 3].

For cases of multiple pulmonary nodules in patients with a single suspected or known primary malignancy, the first differential diagnosis should be multiple lung metastases. Indeed, both clinical and CT pictures in our patient were strongly suggestive of pulmonary metastases. In particular, no CT sign raised suspicion about sarcoidosis due to the lack of lymphoadenomegaly, nodes calcification and pulmonary nodules without an obvious perilymphatic distribution.

For pulmonary lesions, FDG-PET is useful to identify potential malignant lesions and appears particularly useful as an alternative to invasive procedures in the diagnosis of pulmonary metastatic nodules. However, metastatic nodules from extrathoracic malignancies had low sensitivity [5]. On the other hand, FDG-PET uptake from sarcoidosis is non-specific in both intensity and pattern, and can mimic diffuse metastatic disease [6].

Without bronchoscopy to obtain a pathological diagnosis, as in our case, a patient may be treated presumptively and inappropriately for metastatic disease.

Nevertheless, in spite of the extensive diagnostic work-up and the follow-up performed in
Monaldi Arch Chest Dis 2012; 77: 1, 38-39

Key words: Pulmonary metastases, Sarcoidosis, Interferon, Chronic hepatitis.

1 PneumologiaEndoscopia Toracica, 2 Clinica Pneumologica,

3 Sezione di Anatomia Patologica,

Azienda Ospedaliero-

Universitaria di Parma,

4 Scuola

di Specializzazione

in Diagnostica

per Immagini, Università

degli Studi di Parma.

\section{Corrispondence:}

Maria Majori, $M D, P h D$;

Pneumologia-Endoscopia

Toracica, Azienda

Ospedaliero-

Universitaria di Parma,

Padiglione Rasori (25).

Via Rasori 10,

43100 Parma, Italy;

e-mail:mmajori@ao.pr.it 

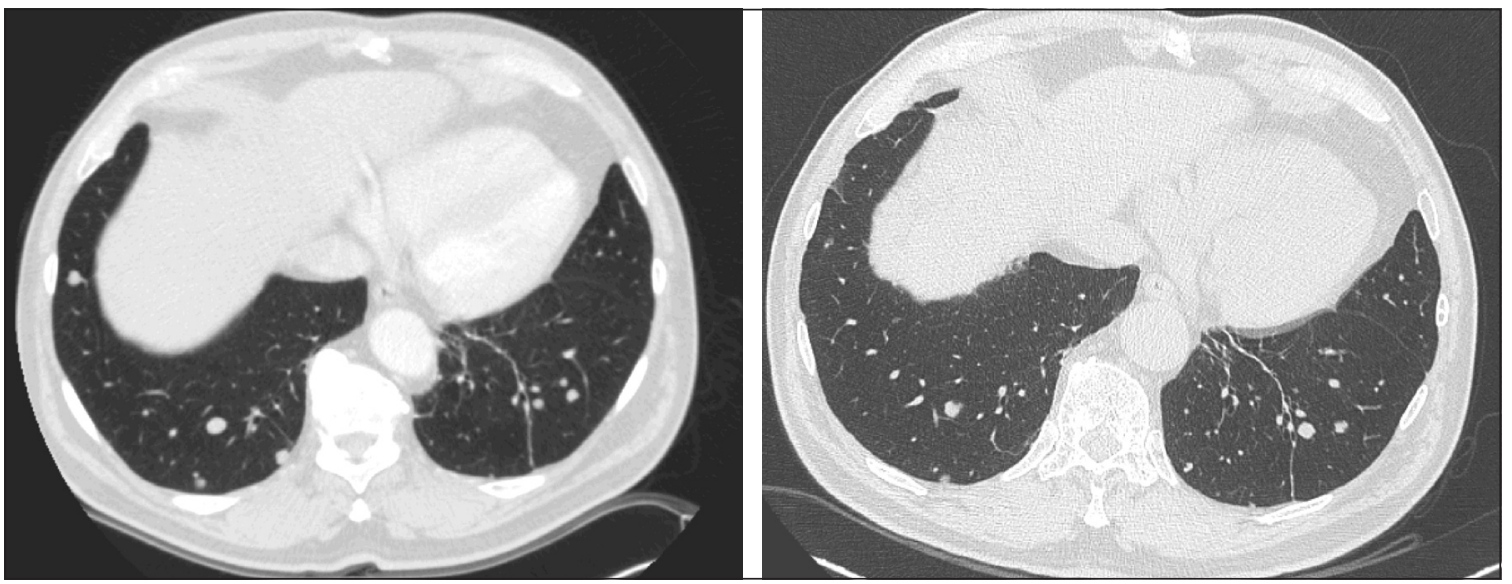

Fig. 1. - Computed tomography scan of the chest showing numerous bilateral nodules ranging in size from few millimeters to 1 $\mathrm{cm}$ in diameter (a) that did not change both in size and number one year later (b).
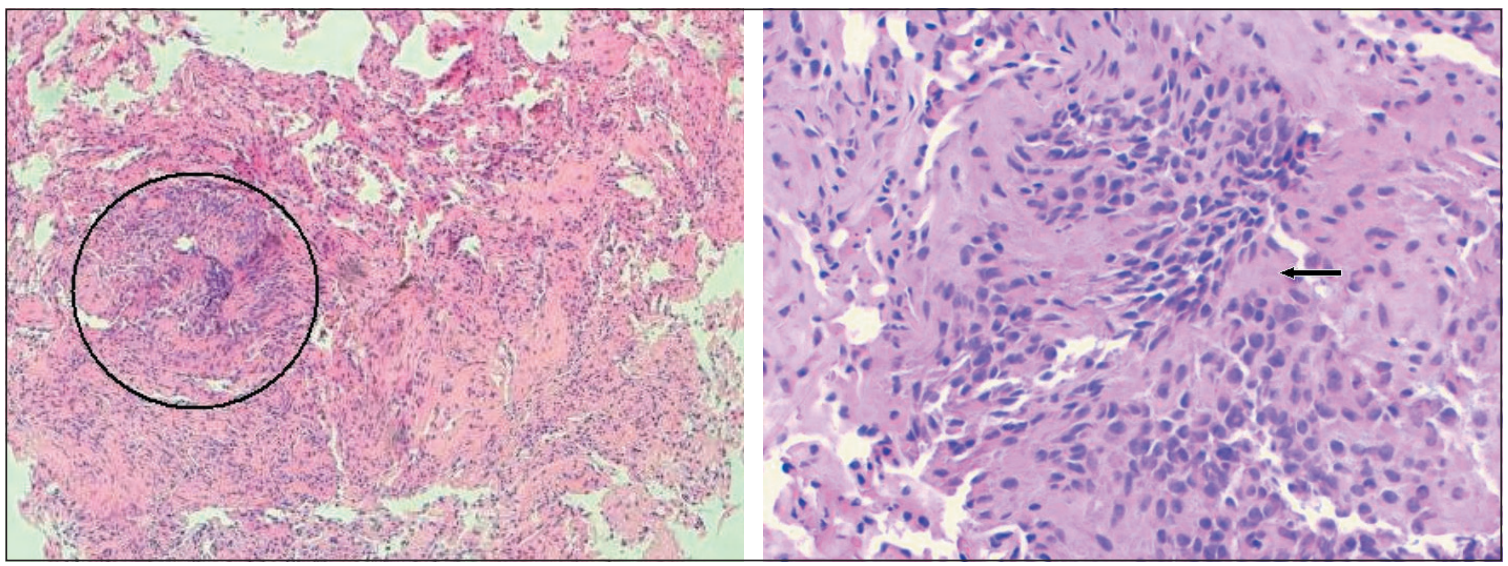

Fig. 2. - Hematoxylin and Eosin staining of the lung specimens obtained by the transbronchial lung biopsy at low magnification $(10 \mathrm{x})$ (a), and at high magnifications $(20 \mathrm{x})(\mathrm{b})$. Noncaseating epithelioid cell granuloma (circle) and multinuclear Langhans giant cells (arrow) were seen.

this patient, a minimal possibility that he had both INF-associated sarcoidosis and pulmonary metastasis still exists because the risk of a sampling error with the tiny specimens obtainable with TBLB is consistent, and only a surgical biopsy including one or more of the nodules seen at CT might have defined their nature beyond any doubt.

\section{References}

1. Abdi EA, Nguyen GK, Ludwing RN, Dickout WJ. Pulmonary sarcoidosis following interferon therapy for advanced renal cell carcinoma. Cancer 1987; 59: 896-900
2. Bobbio-Pallavicini E, Valsecchi C, Tacconi F, Moroni M, Porta C. Sarcoidosis following beta-interferon therapy form multiple myeloma. Sarcoidosis 1995; 12: 140-142.

3. Goldberg HJ, Fiedler D, Webb A, et al. Sarcoidosis after treatment with interferon-alpha: a case series and review of the literature. Respir Med 2006; 100: 2063-2068.

4. Fortes DL, Allen MS, Lowe VJ, et al. The sensitivity of 18 F-fluorodeoxyglucose positron emission tomography in the evaluation of metastatic pulmonary nodules. Eur J Cardiothorac Surg 2008; 34:1223-1227.

5. Koyama T, Ueda H, Togashi K, Umeoka S, Kataoka M, Nagai S. Radiologic manifestations of sarcoidosis in various organs. RadioGraphics 2004; 24: 87-104. 